\title{
Measuring Social Complexity and the Emergence of Cooperation from Entropic Principles. The Collapse of Rapa Nui as a Case Study
}

\author{
O López-Corona ${ }^{1,2,3,}{ }^{*}$, P Padilla $^{2}$, E Schmelkes $^{2}$, JC Toledo-Roy ${ }^{2}$, A Frank $^{2}$, A Huerta ${ }^{5}$, D \\ Mustri-Trejo $^{6}$, K Pérez ${ }^{2}$, A Ruiz ${ }^{2}$, O Valdés ${ }^{2}$, and F Zamudio ${ }^{2}$ \\ ${ }^{1}$ Instituto de Investigación sobre Desarrollo Sustentable y Equidad Social, Universidad \\ Iberoamericana, CDMX, México \\ ${ }^{2}$ Centro de Ciencias de la Complejidad, Universidad Nacional Autónoma de México, CDMX, \\ México \\ ${ }^{3}$ Former: Cátedras CONACyT, Universidad Autńoma Chapingo, Estado de México, México \\ ${ }^{4}$ Instituto de Investigación en Matemáticas Aplicadas y en Sistemas, Universidad Nacional \\ Autónoma de México, CDMX, México \\ ${ }^{5}$ Facultad de Ciencias, Universidad Nacional Autónoma de México, CDMX, México \\ ${ }^{6}$ Maestría en Física, Universidad Veracruzana, Xalapa, Veracruz, México \\ ${ }^{7}$ Grupo de Estadística Social, Universidad Autónoma Chapingo, Estado de México, México \\ All authors contributed equally to this work. \\ *Correspondence: lopezoliverx@otrasenda.org, Tel: +52-55-5950-4339
}

\begin{abstract}
The quantitative assessment of the state and dynamics of a social system is a very difficult problem. This issue is important for both practical and theoretical reasons such as establishing the efficiency of social action programs, detecting possible community needs or allocating resources. In this paper we propose a new general theoretical framework for the study of social complexity, based on the relation of complexity and entropy in combination with evolutionary dynamics to assess the dynamics of the system. Imposing the second law of thermodynamics, we study the conditions under which cooperation emerges and demonstrate that it depends on the relative importance of local and global fitness. As cooperation is a central concept in sustainability, this thermodynamic-informational approach allows new insights and means to assess it using the concept of Helmholtz free energy. We then introduce a new set of equations that consider the more general case where the social system changes both in time and space, and relate our findings to sustainability. Finally we present a model for the collapse of Rapa Nui island civilization in NetLogo. We applied our approach to measure both the entropy production and the complexity of the system and the results support
\end{abstract}

our purpose that sustainability needs a positive entropy production regime which is related to cooperation emergence.

\section{Introduction}

Complexity comes from the Latin plexus, which means interwoven. Something complex is thus difficult to divide, since its interactions are partially responsible for the future of the system 1 .

Given that interactions generate novel information, a reductionist scientific approach has been recognized to be inappropriate for studying complex systems, since it attempts to simplify and separate each component in order to predict its behavior [1. Considering that the information generated by the interactions is not included in its initial and boundary conditions, the predictability of the system is restricted by Wolfram's computational irreducibility $[2$.

Interactions can also be used by components for selforganization, producing global patterns from local dynamics. Furthermore, another major source of complexity is the fact that the interactions themselves may change in time, generating non-stationary state spaces. Therefore, even when a solution can in principle be cal- 
culated, it will become inapplicableat in a futue point if the problem for which it was originally obtained ceases to exist 1].

Just as Lyapunov exponents characterize different dynamical regimes, or temperature represents a statistical average of the kinetic energy of the system, it would be very useful to have global measures for complexity. The interactions between the components in each particular case are difficult to calculate, but complexity measures that represent the type of interactions between them can actually be calculated.

A useful measure of complexity should enable us to answer questions along the follow lines: Is a desert more or less complex than a tundra? What is the complexity of different influenza outbreaks? Which organisms are more complex: predators or prey; parasites or hosts; individuals or society? What is the complexity of different music genres? 3]

As Michaelian (2000) [4] has pointed out, one of the greatest misconceptions preventing the description of biological or social systems from a physical perspective had its origin in the concept of entropy, and the formulation of the second law of Thermodynamics during the original development of the theory of heat engines. In this context, entropy is a measure of the energy that is not available to perform work. Boltzmann later developed Clausius's concept at the microscopic level, relating the entropy of a system to the probability $\left(p_{i}\right)$ of it existing in a macroscopic state, based on the number of equivalent microscopic states that are consistent with the macroscopic state. Here entropy is defined as:

$$
S=-\sum p_{i} \log p_{i}
$$

Entropy, unfortunately, became associated with disorder, the antithesis of complexity. How was it possible that biological or social systems appeared to increase their complexity and reduce disorder, when the second law seems to demand its decrease? One of the most accepted of these explanations establishes that the second law applies only to closed systems, and hence it does not apply directly to biological or social systems which are not closed (they exchange energy, matter and information with their environment). Then, these kind of systems may only reduce their entropy at the expense of the environment, with which they form a closed system.

As discussed by Brooks in his book Evolution as Entropy, the key to solving this apparent paradox is to realize that biological and social systems are nonequilibrium phenomena characterized by an growing phase space and a tendency for realized diversity to lag behind a maximum in entropy. In a physical context, two of the postulated mechanisms for increasing the number of microstates were the expansion of the universe, and the production of elementary particles 6].

Let us consider the diagram in Fig 1 where the vertical

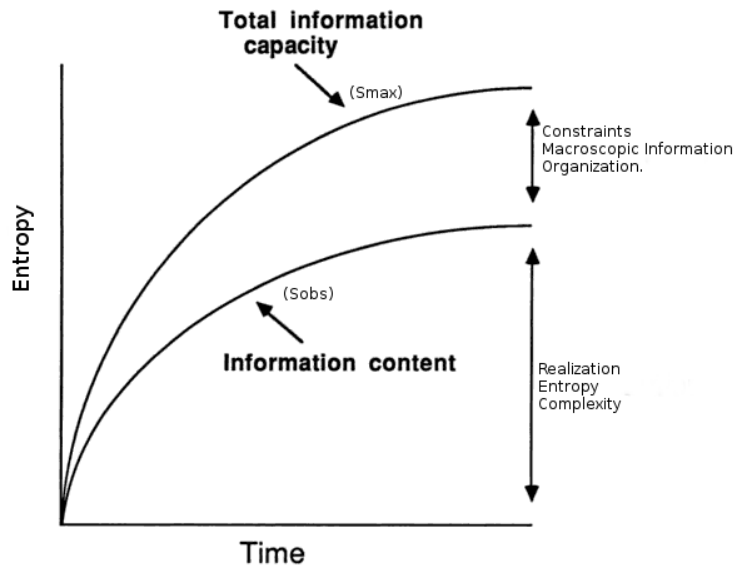

Fig 1. Adapted from "Evolution as Entropy" 7, shows the relation between the total information capacity of a system, associated with the maximum available entropy, and the actual information content of the system after all macroscopic constraints are applied.

axis is a measure of the entropy of the system $(S)$. If the maximum entropy $S_{\max }$ grows with time, then the observed entropy or the final entropy that is attained once all constraints (macroscopical and historical) has been taken into account may also grow.

Considering a more abstract definition of entropy, given a string of characters $X$, composed by a sequence of values or symbols $x$ which follow a probability distribution $P(x)$, information (according to Shannon) is defined as,

$$
I=-\sum p(x) \log p(x)
$$

which has the same functional form as physical entropy. Therefore, in this work we shall talk about entropy $(S)$ and information $(I)$ interchangeably. Then, maximum entropy $S_{\max }$ is the system's total information capacity, while observed entropy $\left(S_{o b s}\right)$ is the actual information content. The macroscopic constraint is macroscopic information and the historical constraint is historicallyexcluded information.

Further, Gershenson and coworkers [5] have proposed that complexity $(C)$ may be directly measured as,

$$
C=a I_{\text {out }}\left(1-I_{\text {out }}\right)
$$

where $a$ is a normalization constant and $I_{\text {out }}$ is the system's information after all computations have taken place. In terms of entropy, it can be written as,

$$
C=a S_{o b s}\left(1-S_{o b s}\right)=a S(1-S)
$$

In this form we may measure the complexity of a system by measuring its entropy. 


\section{Complexity in Evolutionary Dynamics}

At this point, we present a simple computation in the context of game theory as applied to the social sciences that poses some interesting questions. Essentially, we show that if social interactions are described by a game and we compute the associated entropy, then there are some cases in which it will increase monotonically. More precisely, assume for simplicity and without loss of generality that individuals in a society can adopt two strategies $A$ or $B$, and let $x_{A}$ and $x_{B}$ describe the proportion of the population choosing strategy $A$ and $B$ respectively. So we have $x_{A}+x_{B}=1$. Since they are both positive, we can interpret them as probabilities. We assume, in the standard context of game theory, that they satisfy the replicator equations,

$$
\begin{aligned}
& \dot{x}_{A}=\left(f_{A}\left(x_{A}, x_{B}\right)-\phi\right) x_{A} \\
& \dot{x}_{B}=\left(f_{B}\left(x_{A}, x_{B}\right)-\phi\right) x_{B}
\end{aligned}
$$

where the dot indicates the derivative with respect to time, $f_{A}\left(x_{A}, x_{B}\right)$ describes the fitness level assigned to an individual that adopts strategy $A$, and $\phi$ is the average fitness. Now, we may define the entropy of the system as,

$$
S\left(x_{A}, x_{B}\right)=-\left[x_{A} \ln x_{A}+x_{B} \ln x_{B}\right]=-\sum x_{i} \ln x_{i}
$$

and the corresponding entropy production as,

$$
\frac{d S\left(x_{A}, x_{B}\right)}{d t}=-\sum\left[\dot{x}_{i} \ln x_{i}\right]
$$

since $\sum x_{i}=1$ and therefore $\frac{d}{d t} \sum x_{i}=0$. Using the replicator equation we finally get,

$$
\frac{d S}{d t}=-\sum x_{i}\left(f_{i}-\phi\right) \ln x_{i}
$$

\section{Sustainability and Emer- gence of Cooperation}

This is an interesting formulation because the sign of the entropy production depends on whether the fitness of a particular population exceeds the average fitness of the entire population or not. Of course we should be careful about exactly which entropy we are measuring. For all open thermodynamic systems, entropy production has two terms, internal and external, so,

$$
\frac{d S}{d t}=\frac{d S_{i}}{d t}+\frac{d S_{e}}{d t}
$$

All macroscopic systems and processes with an underlying physical basis are subject to definite thermodynamic laws. One of the most important is the second law of thermodynamics, which establishes that the internal production of entropy due to irreversible processes occurring within the system must be positive,

$$
\frac{d S_{i}}{d t}>0
$$

and, for some special cases where external constraints are constant, classical irreversible thermodynamic theory establishes that the system will eventually arrive at a thermodynamic stationary state in which all macroscopic variables, including the total entropy, are stationary in time,

$$
\frac{d S}{d t}=0
$$

and therefore,

$$
\frac{d S_{i}}{d t}=-\frac{d S_{e}}{d t}
$$

implying that,

$$
\frac{d S_{e}}{d t}<0
$$

Maintaining such a system in a stable thermodynamic stationary state requires a continuous flow of entropy into the system.

As the (6) is the total entropy of the game, then the game will evolve to a thermodynamic stationary state when there exists a dominant strategy. Strategic dominance (commonly called simple dominance) occurs when for one player one strategy is better than the other strategies, no matter how his opponents may play. Many simple games can be solved using dominance. The opposite, intransitivity, occurs in games where for one player one strategy may be better or worse than another strategy, depending on how his opponents may play.

Let us assume that $A$ is the dominant strategy and let us write the entropy explicitly,

$$
\frac{d S}{d t}=-\left[x_{A}\left(f_{A}-\phi\right) \ln x_{A}\right]-\left[x_{B}\left(f_{B}-\phi\right) \ln x_{B}\right]
$$

then, since $A$ is dominant, the population with this strategy has to decline over time since its fitness is lower than the average and eventually will disappear. Then, since the population with strategy $B$ is the entire population, the fitness of $B$ and the average fitness are the same and entropy production is zero, reaching a stationary state. This of course means that if the game is representing a social process, this process will require a continuous flow of entropy into the system, which is not sustainable.

Most interesting is that from the thermodynamic stand point, sustainability (understood as the capacity of a system to reach states of greater longevity) is attained by minimizing the Helmholtz free energy, 


$$
F=U-T S
$$

where $T$ is the temperature of the system associated with the internal randomness, and $U$ is the internal energy associated with the energy due to interactions [4]. There are two ways of minimizing Helmholtz free energy, one is by minimizing $U$ and the other is by maximizing $S$. Most of the time the internal energy $U$ is fixed or cannot be controlled deliberately, so the only alternative is maximizing the entropy. As $d S / d t>0$, entropy grows and the system is in a more sustainable configuration. In contrast, when $d S / d t<0$ entropy decreases and the system is moving away from sustainability.

Positive entropy production is only achievable when the average fitness is greater that the local one and that corresponds to cooperative games. Cooperation is essential to sustainability, as might be expected, but now we have a quantitative indicator for measuring how sustainable a system is.

Another interesting aspect of this formulation is that it also clarifies the role of entropy in complexity. We have shown that complexity may be indirectly measured by using the system's entropy, but again we must be very careful in not oversimplifying this with the false idea that complexity and entropy are exactly the same: they are related but they are not the same. The common conception of complexity, prevalent among physicists, is based on the notion of a noninteracting ideal gas. It is only in this case that a direct association with entropy has some legitimacy. In the real world, interactions are an integral part of the ordering of material particles. In fact, as pointed out by Michaelian (2000) 4, a definition of order had been given long ago by Helmholtz in his formulation of free energy (15). Increasing the entropy in 15) decreases the free energy of the system. In this scheme, order has a very natural description, and increasing the order of the system makes it more stable, lasting longer in time, more sustainable. This is true in the ideal gas approaching equilibrium, the crystallization of matter, or the evolutionary dynamics of a community of interacting biological or social systems.

Then, the association between information and entropy is tricky because complexity is measured as information after all the computations of the system has been carried out. This information, of course is partially comprised of interactions and thus the entropy to be used must include them. The entropy that appears in the Helmholtz free energy expression is thus appropriate. This allows us to make a direct connection with the entropy production calculated from the replicator equation and, as Michaleian [4 suggests, identify sustainability with Helmholtz free energy minimization. Then, fitness is a measure of this capacity for minimization.

Until now, in this first approximation, we have only worked with the temporal dimension and not even fully so, because we have assumed that interactions do not change over time. But, of course, in social systems interactions may evolve with time; for example two agents that used to cooperate may suddenly stop cooperating (or vice-versa), changing the form of the interaction and thus the entropy of the system as Axelrod proved [8]. Even more, the spatial dimension plays a key role in the emergence of cooperation 9 so we must understand how it contributes to entropy.

Let us begin by considering only the spatial contribution to entropy with non-time-dependent interactions in a system of $n$ replicator equations in a space defined by a set of $r_{j}$ coordinates as,

$$
\frac{\partial x_{i}\left(r_{j}, t\right)}{\partial t}=x_{i}\left(f_{i}\left(x_{1}, \ldots, x_{i}, \ldots, x_{n}\right)-\phi\right)
$$

We assume some basic probabilistic properties for these equations as if $0 \leq x_{i}\left(r_{j}, 0\right) \leq 1$ then for every subsequent time period $0 \leq x_{i}\left(r_{j}, t\right) \leq 1$. In the same way if $\sum x_{i}\left(r_{j}, 0\right)=1$ then for every subsequent time period $\sum x_{i}\left(r_{j}, t\right)=1$.

Under these considerations, the first approximation for considering a spatial contribution would be introducing a diffusive term in the replicator equations,

$$
\frac{\partial x_{i}\left(r_{j}, t\right)}{\partial t}=\epsilon_{i} \Delta x_{i}+x_{i}\left(f_{i}-\phi\right)
$$

where $\epsilon_{i}$ is a diffusion coefficient and $\Delta$ is the laplacian operator.

Now, an interesting question arises: under which conditions is the rate of change of total entropy, $\frac{d}{d t} \int_{\Omega} S\left(r_{j}, t\right) d r_{j}$, is larger than zero, so the second law of thermodynamics holds?

We have that,

$$
\frac{d}{d t} \int_{\Omega} S\left(r_{j}, t\right) d r_{j}=\int_{\Omega} \frac{\partial}{\partial t} S\left(r_{j}, t\right) d r_{j}
$$

and using the definition of entropy, $S=-\sum x_{i} \ln x_{i}$,

$$
\frac{d}{d t} \int_{\Omega} S\left(r_{j}, t\right) d r_{j}=-\sum\left[\int_{\Omega} \frac{\partial}{\partial t}\left(x_{i} \ln x_{i}\right) d r_{j}\right]
$$

Applying the derivative and using the replicator equation,

$$
\begin{array}{r}
\frac{d}{d t} \int_{\Omega} S\left(r_{j}, t\right) d r_{j}=-\int_{\Omega}\left\{\frac{\partial}{\partial t}\left(\sum x_{i}\left(r_{j}, t\right)\right)\right. \\
\left.+\sum\left[\epsilon_{i} \Delta x_{i}+x_{i}\left(f_{i}-\phi\right)\right] \ln x_{i}\right\} d r_{j}
\end{array}
$$

and using the normalization condition $\sum x_{i}\left(r_{j}, t\right)=1$ it turns out that $\frac{\partial}{\partial t}\left(\sum x_{i}\left(r_{j}, t\right)\right)=0$, and so, 


$$
\begin{array}{r}
\frac{d}{d t} \int_{\Omega} S\left(r_{j}, t\right) d r_{j}=-\sum \int_{\Omega}\left\{\left[\epsilon_{i} \Delta x_{i}\right.\right. \\
\left.\left.+x_{i}\left(f_{i}-\phi\right)\right] \ln x_{i}\right\} d r_{j}
\end{array}
$$

and finally,

$$
\begin{aligned}
\frac{d}{d t} \int_{\Omega} S\left(r_{j}, t\right) d r_{j} & =-\sum \int_{\Omega} \epsilon_{i} \Delta x_{i} \ln x_{i} d r_{j} \\
& -\sum \int_{\Omega} x_{i}\left(f_{i}-\phi\right) \ln x_{i} d r_{j}
\end{aligned}
$$

Now the sign of the entropy production is, in part, defined by the sign of $\left(f_{i}-\phi\right)$ in the second integral but, also, by the spatial term in the first. Focusing on the spatial integral and using the Divergence Theorem we obtain that,

$$
\begin{array}{r}
\int_{\Omega} \epsilon_{i} \Delta x_{i} \ln x_{i} d r_{j}=-\int_{\Omega} \nabla x_{i} \cdot \nabla\left(\ln x_{i}\right) d r_{j} \\
+\int_{\partial \Omega} \ln x_{i} \frac{\partial x_{i}}{\partial r_{j}} d \sigma
\end{array}
$$

where an inflow of probability to the region $\Omega$ is stablished when $\frac{\partial x_{i}}{\partial r_{j}}>0$ and an outflow from the region occurs when $\frac{\partial x_{i}}{\partial r_{j}}<0$. Considering that $\nabla \ln x_{i}=\frac{1}{x_{i}} \nabla x_{i}$ then we have that $\int_{\Omega} \epsilon_{i} \Delta x_{i} \ln x_{i} d r_{j}>0$ for positive probability flux.

These results imply that if $\phi>f_{i}$ and the probability flux is positive then we recover the second law of thermodynamics $\frac{d S_{i}}{d t} \geq 0$.

Now let's consider a more general case where the interaction may change in time and space. For this instead of the replicator equation, we start by using the López-Padilla equations 10 .

$$
\frac{\partial x_{i}\left(r_{j}, t\right)}{\partial t}=\operatorname{div}\left[e_{i}\left(r_{j}, t\right) \nabla x_{i}\left(r_{j}, t\right)\right]
$$

where $x_{i}$ is the probability of finding a player in the position $r_{j}$ at the time $t$; and $e\left(r_{j}, t\right)$ is the corresponding strategy that obeys the equation,

$$
\begin{array}{r}
\frac{\partial e_{i}\left(r_{j}, t\right)}{\partial t}=- \\
+\nabla^{2}\left[\phi\left(r_{j}, t\right) e_{i}\left(r_{j}, t\right) e_{i}\left(r_{j}, t\right)\right]
\end{array}
$$

As before, we want to calculate entropy production,

$$
\frac{d}{d t} \int_{\Omega} S\left(r_{j}, t\right) d r_{j}=-\sum\left[\int_{\Omega} \frac{\partial}{\partial t}\left(x_{i} \ln x_{i}\right) d r_{j}\right]
$$

but now we use the new set of equations.

$$
\begin{array}{r}
\frac{d}{d t} \int_{\Omega} S\left(r_{j}, t\right) d r_{j}= \\
-\sum \int_{\Omega}\left\{d i v\left[e_{i}\left(r_{j}, t\right) \nabla x_{i}\left(r_{j}, t\right)\right] \ln x_{i}\right\} d r_{j}
\end{array}
$$

This new expression for entropy production considers the complete contributions of space and interactions, but now the analysis for determine the conditions in which entropy production is monotonous is not a trivial one, and it will depend on the nature of the interactions represented by the strategies $e\left(r_{j}, t\right)$.

\section{The Collapse of Rapa Nui Is- land, a case of study}

Easter Island, also called Rapa Nui by its inhabitants, is a place so remote that its civilization never contacted any other human group from the time of its settlement (c. $000=1200$ A.D. 11]) to the year the first Europeans arrived in the island (1722 A.D.). This civilization flourished for for several centuries, developing an impressive economy and culture. However, between 1600 and 1800 A.D. the Rapa Nui civilization underwent ecological, societal, and economic collapse. From a high watermark of around 13000 people [12, the population of the island crashed to 3000 in less than two hundred years. The reason for this collapse is as yet unknown, but the most accepted hypothesis is that excess deforestation of the island caused a food shortage that led to internal warfare and an eventual radical decline in population and material culture 13.

The population of Rapa Nui survived and thrived on a few sources of food. They grew taro and bananas, bred Polynesian chickens, fished tuna and dolphins and gathered shallow-water mollusks. Though the island has been barren since we have record of it, pollen records offer strong evidence that the island was once completely covered by palm trees 14 . The Rapa Nui people must have cut down these trees to clear land for agriculture, and to build vessels to fish in the open ocean [13].

The monumental stone statues of the island, called moai, were built during this period of growth and prosperity. However, at some point in the 17th century, obsidian spearheads begin to appear in the archaeological records 15. After a thousand years of peace, war broke out between the clans of the island. When the island was first contacted by Europeans, the great statues were all standing, but each successive European visitor to the island during the 18 th and early $19^{\text {th }}$ centuries records more and more toppled moai. By 1850 , all statues had been toppled, and the island had shifted to a different religion and form of government. 


\section{IV.1 The Rapa Nui Model}

The fact that this self-contained ecosystem harbored a population that had no contact with any other civilization, and therefore no trade, migration or military activity, makes it a very interesting natural economic experiment. This model was first developed in Elisa Schmelkes undergraduate dissertation to create a simulation of the island that could bring us closer to understanding what caused the collapse of Rapa Nui. The model uses system dynamics and two interacting stocks of population and resources to create a simple simulation of a coupled population-resource system.

In this model, the variable that determines the class of outcome obtained in the simulation (stability, oscillation or collapse) is technology, defined here as access to the scarcest resource in the system. This variable, which relates to labor productivity, affects the speed at which population grows, and hence the speed with which it can recover from ecological crashes. When a population has low access to its scarcest (renewable) resource, it cannot grow fast enough to erode it, and reaches ecological equilibrium. At intermediate levels of access, when the population starts eroding the resource, as population grows resource levels fall, allowing the resource to recover and oscillate. Finally, when a population has high access to its scarcest resource, it grows too fast at a rate that exceeds its capacity for recovery. Once this happens, it's too late for the resource to recover, and they both collapse.

In order to further study the dynamics of the system, the model was later extended into a spatial agent-based model with several different clans. Each clan is an agent and grows organically according to the rules of the earlier model. The finite area of the island is subdivided into patches that contain resources, determining the carrying capacity. Each of the patches can be harvested for resources, and its resources renovate according to the erosion of the entire island. Each clan initially owns a single patch of land. As the clans grow and require more food, they begin acquiring more land by occyping nearby patches, until the entire island is settled.

We introduced competition by including in this expanded model several strategies that can be taken by each of the agents. Loosely following the model for bacterial coexistence proposed by [16] Kerr et al. (2002), each of the agents (i.e. clans) can use their human resources to either (a) be more productive and grow faster, (b) be more aggressive and take other clans' patches more easily, or (c) defend itself from attacks by other clans. Aggression is considered more expensive than resistance and thus imposes a heavier resource toll, generating Rock-Paper-Scissors-like dynamics. Further, each turn the clans see if their strategy has worked by checking whether they have grown in the past two turns. If they haven't, they randomly choose another strategy.
This generates interesting new dynamics. Initially, the clans that invest their resources into growth, tend to dominate the landscape, and then engage in battle with the aggressive clans. At mid to high levels of technology, the aggressive clans usually win out and dominate in the end. However, at lower levels, they mostly coexist as they reach an equilibrium with their environment.

By applying the methods of analysis proposed in this paper to the dynamics of the Rapa Nui model, we shown (see Fig. 2) that while the total population and resources curves do not intersect, the system is in a steady state $(d S / d t=0)$ with virtually homogeneous population growth for all clans. It seems that the clan with the greatest initial growth will go thorough a more drastic population drop and will face collapse earlier. When the population and resources curves intersect, the systems lose stationarity $(d S / d t \neq 0)$. At this point for the sustainability experiment (low levels of fragility) where cooperation emerges and all clans coexists, negative entropy production takes place but the systems reacts to it with a positive entropy flow, and after a short oscillation in entropy production the system converges (from positive entropy production values) to a new stationary state. Initially, we observe a decrease of complexity while population is growing and before the curves intersect. Then, after intersection, the system responds with soft complexity gain and subsequent stabilization. On the other hand, when we have high values of fragility (the collapse experiment) after the loss of stationarity, the system enters negative entropy production, as with the sustainability experiment. Then the system also responds with flows of positive entropy. However, in contrast with the previous case, the system does not converge to stationarity but remains in a mode of negative entropy production. Negative entropy production grows in a step fashion with positive entropy production deltas whenever a clan collapses. When only one (or no) clan subsists, the system reaches a new non-cooperative stationary state. From the complexity perspective, every time a clan collapses there is a jump in the complexity growing process and the final complexity value in the collapse experiment is lower that the one reached in the sustainability experiment.

\section{Discussion and Conclusions}

Our proposed new equation is very general and for the first time (to our knowledge) takes into account the contributions of both interactions and space, as well as their time dependence. In the more restricted case where the spatial component is not taken into account, we have analytically shown that the emergence of cooperation is a consequence of entropic principles and that it may be induced by controlling the difference between global and local fitness. This mathematical 


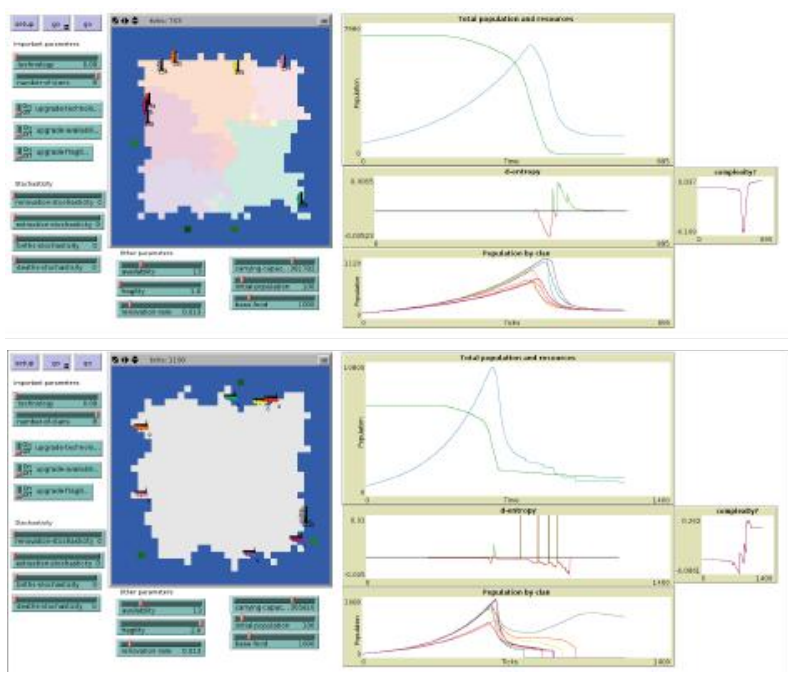

Fig 2. Examples of NetLogo Rapa Nui experiments under different leves of fragility to induce sustainability or collapse. The upper sub-figure is the low fragility experiment where sustainability emerges and higher values of complexity are reached. The bottom sub-figure is the high fragility experiment that leads to a civilization collapse in which only one clan prevails and correspond to lowest values of complexity

conclusion is supported by the results measured in the Rapa Nui Model, in which collapse takes place only when a negative entropy production gets established, meaning that local fitness is greater than global fitness. Further, when the system dynamics is dominated by positive entropy production, cooperation emerges (clans coexist) and greater values of complexity are reached once the system returns to stationarity. Thus the hypothesis that sustainability is related to positive entropy production regimes, corresponding to greater values of complexity, is confirmed at least for this model. This seems to us very important, because it opens the door to the construction of sustainability indicators based on entropy and complexity measurements.

\section{Acknowledgements}

OLC thanks Fondo Capital Semilla at Universidad Iberoamericana, former support of Catedras CONACyT and to SNI program with number 62929.

\section{References}

1. Gershenson, C. The implictions of interactions for sciende and phylosophy. Found. Sci. 18.4 (2013): 781-790.
2. Wolfram, S. A new kind of science. Vol. 5. Champaign: Wolfram media (2002).

3. Fernandez, N., Maldonado, C.,Gershenson, C., Information measures of complexity, emergence, self-organization, homeostasis, and autopoiesis. Guided Self-Organization: Inception. Springer (Berlin Heidelberg) (2014): 19-51.

4. Michaelian, K., A Physical Basis of Evolution and Speculation on an Evolutionary Basis of Physics Chapter in Topics in Contemporary Physics, 195210, Ed. J. Heras, ISBN 970-18-3700-2 (2003).

5. Gershenson, C., Fernandez, N. Complexity and information: Measuring emergence, selforganization, and homeostasis at multiple scales. Complexity 18.2 (2012): 29-44.

6. Frautschi, Steven. Entropy in an expanding universe. Science 217.4560 (1982): 593-599.

7. Brooks, D. R., Wiley, E. O., and Brooks, D. R. Evolution as entropy. Chicago: University of Chicago Press (1988).

8. Axelrod, Robert M. The evolution of cooperation. Basic books (2006).

9. Nowak, Martin A. Evolutionary dynamics. Harvard University Press (2006).

10. López-Corona, O., et al. Playing with models and optimization to overcome the tragedy of the commons in groundwater. Complexity 19.1 (2013): 9-21.

11. Hunt, T. L., and Lipo, C. P. Late colonization of Easter island. Science, 311.5767 (2006): 16031606 .

12. Brandt, Gunnar, and Agostino Merico. The slow demise of Easter Island: insights from a modeling investigation. Frontiers in Ecology and Evolution 3 (2015): 13 .

13. Flenley, J., and Bahn, P. The Enigmas of Easter Island. Oxford University Press, UK (2003).

14. Diamond, Jared. Collapse: How societies choose to fail or succeed. Penguin, (2005).

15. Bahn, Paul G., and John Flenley. Easter Island, earth island. Thames and Hudson, (1992).

16. Kerr, Benjamin, et al. Local dispersal promotes biodiversity in a real-life game of rock-paper-scissors. Nature 418.6894 (2002): 171174. 
17. Davis, Brent, and Dennis J. Sumara. Challenging images of knowing: Complexity science and educational research. International Journal of Qualitative Studies in Education 18.3 (2005): 305-321.

18. Davis, Brent, and Dennis J. Sumara. Complexity and education: Inquiries into learning, teaching, and research. Psychology Press, 2006.

19. Frei, Regina. A complex systems approach to education in Switzerland. Advances in artificial life, ECAL 2011. Proceedings of the eleventh European conference on the synthesis and simulation of living systems networks. 2011.

20. Morrison, Keith. Complexity theory and education. APERA Conference, Hong Kong. 2006.

21. Lämmer, S., Helbing, D. Self-control of traffic lights and vehicle flows in urban road networks. Journal of Statistical Mechanics: Theory and Experiment 2008.04 (2008): P04019. 